\title{
IV. Zusammenfassende Schlußbetrachtung
}

Gemäß dem Gesamtsansatz der Studie wird im folgenden der Versuch unternommen, die Wirkungszusammenhänge von ökonomischer Entwicklung, sozialen Veränderungen und staatlich-verbandlichen Interventionen zu bestimmen. Dabei soll zunächst die Wechselwirkung zwischen ökonomischer und sozialer Entwicklung beleuchtet werden. In einem zweiten Schritt wird die Frage erörtert, inwieweit die politischen Steuerungsversuche die sozialökonomischen Basisprozesse beeinflußten. Abschließend werden die Ergebnisse des dritten, überwiegend politikgeschichtlichen Untersuchungsteils im Zusammenhang dargestellt.

Im Untersuchungszeitraum setzte sich der säkulare ökonomische Strukturwandel des gewerblichen Mittelstandes beschleunigt fort. Nachdem es in der unmittelbaren Nachkriegszeit aufgrund der desolaten Beschäftigungssituation zu einer „Flucht in die Selbständigkeit" gekommen war, ging seit Beginn der 50er Jahre die Betriebsdichte und damit auch die Zahl der Betriebe signifikant zurück. Die Wettbewerbsintensität stieg insbesondere in den unteren Betriebsgrößenklassen spürbar an. Der Wandel der Marktverhältnisse führte dazu, daß die Konkurrenzfähigkeit leistungsschwacher Betriebe abnahm. Zu den Opfern dieser Entwicklung zählten vor allem traditionelle Kleinstbetriebe (1 bis 4 Beschäftigte). Etwa ab Mitte der 50er Jahre ist die Ausdünnung der handwerklichen Kleinstbetriebe auch vor dem Hintergrund des zunehmend attraktiver werdenden Arbeitnehmerstatus (steigendes Einkommen, Verbesserung der sozialen Sicherung, Freizeitzuwachs) zu sehen. Der Wechsel in eine abhängige Berufsstellung stellte allerdings nur für jüngere Handwerker eine ernstzunehmende Alternative zur selbständigen Berufsausübung dar. Für die älteren Betriebsinhaber bedeutete die Betriebsaufgabe meist den unwiederbringlichen Verlust ihrer Existenzbasis. Um dies zu verhindern, führten sie ihre Betriebe häufig selbst dann weiter, wenn die Einnahmen marginal waren. Ohne diese sozialen Sicherungsprobleme wäre der handwerkliche Betriebsbestand vermutlich noch schneller gesunken. Auch im Einzelhandel dürfte die Bereitschaft, den Betrieb stillzulegen, mit steigendem Alter abgenommen haben. Allerdings ist zu betonen, daß im Einzelhandel - im Gegensatz zum Handwerk - der Betriebsbestand in den 50er Jahren nicht schrumpfte, sondern leicht zunahm. Hier kam es erst in den 60 er Jahren zu einem massenhaften Ausscheiden der Kleinstbetriebe vom Markt.

Welche sozialen Konsequenzen zog der Betriebsrückgang im Handwerk nach sich? Der wohl augenfälligste Effekt bestand darin, daß der Anteil der Handwerker an der Gesamtbevölkerung abnahm. Die Sozialgruppe der Handwerker schrumpfte also. Diese Tatsache allein ist indes wenig aussagekräftig. Erst wenn man sie in Verbindung mit dem sich in den 50er Jahren beschleunigenden Betriebsgrößenwachstum sieht, gewinnt sie an Gewicht. Der mit dem Betriebsrückgang einhergehende durchschnittliche Anstieg der Beschäftigtenzahl pro Betrieb resultierte in erster Linie aus der zunehmenden räumlichen Konzentration der Nachfrage und dem gleichzeitigen Anstieg der Kaufkraft. Mit der Betriebsgröße nahm auch das Durchschnittseinkommen der Betriebsinhaber zu.

Im Einzelhandel manifestierte sich das Betriebsgrößenwachstum weniger in der $\mathrm{Zu}$ nahme der Beschäftigtenzahl pro Betrieb als in der Erhöhung des betrieblichen Umsatzvolumens. Infolge dieser Entwicklung stieg das Einkommen eines Großteils der Inha- 
ber von kleinen Einzelhandelsbetrieben signifikant an. Insgesamt wird man feststellen können, daß der Anteil der proletaroiden Selbständigen, die im allgemeinen keine oder nur wenige familienfremde Arbeitskräfte beschäftigten, im gewerblichen Mittelstand spürbar abnahm. Der Typus des Kleinunternehmers gewann an Boden. Diese Entwicklung führte indes nicht dazu, daß sich innerhalb der mittelständischen Berufsgruppen ein einheitlicher Lebensstandard herausbildete. Die absoluten Einkommensunterschiede waren nach wie vor beträchtlich. Erhebliche Differenzen lassen sich auch in bezug auf die Vermögensverhältnisse und die Konsumgüterausstattung nachweisen, wobei die Polarisierung im Kleinhandel stärker ausgeprägt war als im Handwerk. Im Vergleich zur Zwischenkriegszeit hatten sich die berufsgruppeninternen Ungleichheiten im Zuge der kollektiven Einkommenserhöhung nach „oben“ verschoben. Die Spaltung der mittelständischen Betriebsinhaber in proletaroide Selbständigenexistenzen auf der einen und Inhaber prosperierender Betriebe auf der anderen Seite schwächte sich in den 50er Jahren deutlich ab. Die berufsgruppeninterne Ungleichheitslinie verlief nicht mehr zwischen Arm und Reich. Gegen Ende des Untersuchungszeitraums standen sich vielmehr Handwerker mit bescheidenem Wohlstand und (relativ) wohlhabende Kleinunternehmer gegenüber. Die Einkommensunterschiede zwischen den Handwerkszweigen prägten sich im Untersuchungszeitraum schärfer aus. Die Bedeutung der Branchenzugehörìgkeit für den Lebensstandard nahm also zu. Die Herausbildung eines branchenübergreifenden Gemeinschaftsbewußtseins dürfte hierdurch erschwert worden sein.

Der enge Zusammenhang zwischen Arbeits- und Familienleben bildete seit jeher ein Spezifikum der mittelständischen Lebensführung. Dies änderte sich auch in den 50er Jahren nicht. In ökonomischer Hinsicht trat zwar der Stellenwert der Familie für die im Betrieb erbrachte Arbeitsleistung angesichts der durchschnittlich wachsenden Beschäftigtenzahl pro Betrieb zunehmend in den Hintergrund. Aus sozialgeschichtlicher Perspektive ist jedoch ein gegenläufiger Trend zu konstatieren, zumal in bezug auf das Handwerk, in dem sich die personelle Verflechtung zwischen Familie und Betrieb sogar verdichtete.

Was die „objektiven“ Lebensbedingungen betrifft, veränderten sich die Ungleichheitsrelationen zwischen den selbständigen Mittelständlern und den Arbeitnehmern, gemessen an den Mittelwerten der jeweiligen Berufsgruppen, nur unwesentlich. In einigen Bereichen verschärfte sich sogar die Differenz zwischen Arbeitnehmern und mittelständischen Gewerbetreibenden (vor allem PKW-Besitz/ Arbeitszeit). Letztlich wird man allerdings feststellen müssen, daß die soziale Bedeutung der untersuchten Ungleichheitsdimensionen abnahm. Dies hing vor allem mit der gesamtgesellschaftlichen Erhöhung des materiellen Wohlstandsniveaus sowie mit der Erosion traditioneller Milieugrenzen zusammen. Letztere führte zu einer allmählichen Auflösung klassengesellschaftlicher Deutungsmuster. Der Mittelstand wurde zwar auch gegen Ende des Untersuchungszeitraums noch als soziale Formation wahrgenommen, die Mittelstandszugehörigkeit büßte jedoch insofern an sozialer Relevanz ein, als die Abgrenzung gegenüber den Arbeitern nicht mehr so dringlich schien und es auch innerhalb der mittelständischen Berufsgruppen tendenziell zu einer Versachlichung der Sozialbeziehungen kam. So traten die nichtökonomischen Funktionen der lokalen Mittelstandsverbände zurück. Die noch zu Beginn der 50er Jahre in Mittelstandskreisen weitverbreitete Vorstellung, eine soziale Mittellage einzunehmen und als „Puffer“ zwischen den Klassen eine zentrale gesellschaftliche Funktion innezuhaben, verlor ebenfalls sukzessive an Bedeutung. 
Die Analyse der Mobilitätsprozesse deutet darauf hin, daß die soziale Abgeschlossenheit der mittelständischen Berufsgruppen eher zu- als abnahm. Öffnungstendenzen im Sinne einer Heterogenisierung der Herkunftsstruktur sind jedenfalls auf der Grundlage des ausgewerteten statistischen Materials nicht erkennbar. Es dominierte nach wie vor die Selbstrekrutierung, wobei sich diese stärker auf die einzelnen Branchen denn auf die Berufsgruppen als ganze oder gar auf den gewerblichen Mittelstand bezog. Arbeiterkinder blieben in den mittelständischen Berufsgruppen deutlich unterrepräsentiert. Dieser Befund läßt sich mit den unterschiedlichen Sozialisationsbedingungen von Arbeiterund Selbständigenkindern erklären. Es gibt Indizien dafür, daß in mittelständischen Familien die Selbständigkeit als Ziel der beruflichen Laufbahn einen höheren Stellenwert einnahm als in Arbeiterfamilien. Des weiteren ist zu berücksichtigen, daß der Aufstieg zum selbständigen Handwerksmeister bzw. Ladeninhaber insofern schwieriger wurde, als die für die Errichtung oder Übernahme eines konkurrenzfähigen Betriebes erforderliche Kapitalsumme im Untersuchungszeitraum deutlich anstieg. Dies hing zum einen mit dem Betriebsgrößenwachstum und zum anderen mit der sich vor allem in der zweiten Hälfte der 50er Jahre beschleunigenden technischen Rationalisierung und der damit verbundenen Erhöhung der Kapitalintensität zusammen. Unter diesen Bedingungen dürfte es den Mittelstandskindern leichter gefallen sein, den Schritt in die Selbständigkeit zu tun, als den Töchtern und Söhnen von Arbeitern.

Die sozialgeschichtlichen Wirkungen des branchenstrukturellen Wandels sind in der vorliegenden Studie nur ansatzweise untersucht worden. Immerhin erscheint es bemerkenswert, daß im Untersuchungszeitraum gerade jene Handwerkszweige an Boden verloren, die über Jahrhunderte hinweg das Gesicht des Handwerks geprägt hatten. So büßten neben den Bekleidungshandwerken und den Landhandwerken, die zum Teil ganz von der Bildfläche verschwanden, auch die Holzhandwerke ihre einstmals zentrale Stellung in der Handwerkswirtschaft ein. Die Schrumpfung der Bekleidungshandwerke trug maßgeblich dazu bei, daß der ohnehin geringe Anteil der Frauen unter den selbständigen Handwerken in den 50er Jahren zurückging. Auf Expansionskurs befanden sich insbesondere die modernen technischen Handwerksbranchen, die über keine nennenswerte berufsständische Tradition verfügten.

Ein wesentliches Kennzeichen des sozialökonomischen Strukturwandels im gewerblichen Mittelstand ist in der Verschiebung der Berufsinhalte der Betriebsinhaber und ihrer Mitarbeiter zu sehen. So nahm im Handwerk die Handelstätigkeit spürbar zu. Dieser Trend ging in vielen Branchen mit einer zunehmenden Spezialisierung einher. Die Produktionsfunktion des Hand werks gewann in den 50er Jahren an Bedeutung. Der Umsatzanteil aus Reparaturleistungen war dagegen leicht rückläufig. Die Technisierung und - in einem spezifischeren Sinne - die Maschinisierung und Motorisierung setzten sich in der zweiten Hälfte der 50er Jahre beschleunigt fort. Im allgemeinen wirkte sich der Einsatz neuer technischer Hilfsmittel und Verfahren als Arbeitserleichterung aus. Im Großteil der modernen technischen Branchen erhöhten sich im Zuge dieser Entwicklung die theoretischen und praktischen Anforderungen. Die These, durch Maschinisierung und Standardisierung seien traditionelle Produktionsmethoden unterhöhlt und somit der Trend zur Dequalifizierung der Handwerksarbeit verstärkt worden, trifft nur auf einen Teil der Handwerkszweige zu. Obwohl die Industrie im Untersuchungszeitraum verstärkt als Abnehmer handwerklicher Produkte und Leistungen auftrat, wurde hierdurch die Unabhängigkeit des Handwerks nicht nennenswert beeinträchtigt. Der Typus des reinen Zulie- 
ferbetriebes, der nur für wenige großindustrielle Abnehmer tätig war, stellte auch gegen Ende des Untersuchungszeitraums die Ausnahme dar. Im Einzelhandel waren die durch die ökonomische Entwicklung bedingten Veränderungen des Tätigkeitsprofils einschneidender als im Handwerk. Im Zuge der absatztechnischen Rationalierung, die sich Ende der 50er Jahre auf breiter Basis durchzusetzen begann und die vor allem durch die Einführung der (Teil-)Selbstbedienung gekennzeichnet war, verloren einstmals zentrale Aufgabenbereiche an Bedeutung (Verkaufsgespräch, Verkaufsvorbereitung usw.). Zum Wandel des Tätigkeitsprofils trugen außerdem die im Untersuchungszeitraum rasch expandierenden Einkaufsgenossenschaften und freiwilligen Ketten bei. Die Mitglieder dieser als Modernisierungsagenturen fungierenden Großzusammenschlüsse waren von einer zunehmenden Funktionsausgliederung betroffen. So traten traditionelle Aufgabenbereiche wie z. B. die Warenbeschaffung und die Preisfestsetzung zunehmend in den Hintergrund. Die wirtschaftliche Autonomie der Betriebsinhaber wurde spürbar eingeschränkt. Dem stand allerdings eine bemerkenswerte Erhöhung der betrieblichen Leistungsfähigkeit gegenüber. Diejenigen Einzelhändler, die an überkommenen Geschäftsführungsmethoden festhielten, gerieten immer mehr in die Defensive. Die genannten Entwicklungen wirkten sich im Lebensmitteleinzelhandel, der mit Abstand größten Einzelhandelsbranche, am nachhaltigsten aus. Daneben gab es allerdings auch Einzelhandelszweige, in denen der traditionelle Facheinzelhandel keinem nennenswerten Modernisierungsdruck ausgesetzt war.

Die ökonomische Wertstruktur der Kleingewerbetreibenden stellte zweifellos ein retardierendes Moment in der wirtschaftlichen Entwicklung der mittelständischen Betriebe dar. Wie sozialwissenschaftliche Erhebungen aus den späten 50er und frühen 60er Jahren belegen, stand eine starke Minderheit der Betriebsinhaber in Handwerk und Einzelhandel kapitalistisch-marktwirtschaftlichen Prinzipien äußerst kritisch gegenüber. Dies äußerte sich unter anderem in ablehnenden Stellungnahmen gegenüber dem Wettbewerb, der freien Preisbildung, der Gewinnmaximierung sowie der technischen Innovation. Das primäre Ziel des Wirtschaftens bestand für viele Handwerker und Einzelhändler nach wie vor in einer auskömmlichen Lebenshaltung und nicht in der betrieblichen Expansion. Preisabsprachen galten als legitim und wurden vielerorts praktiziert. Vormarktliche Einstellungen waren insbesondere unter den Inhabern kleinerer Betriebe weit verbreitet, also in jener Gruppe, die im Zuge des ökonomischen Strukturwandels vergleichsweise stark schrumpfte. Man wird also davon ausgehen können, daß Anfang der 50er Jahre der Anteil der Traditionalisten höher war, als es die ein Jahrzehnt später angestellten Untersuchungen erkennen lassen. Dies erscheint auch deshalb wahrscheinlich, weil im Verlauf der 50er Jahre der Anteil der modernisierungswilligeren jüngeren Alterskohorten unter den Betriebsinhabern zunahm.

Die Modernisierung des ökonomischen Verhaltens hing nicht nur von der Anpassungsbereitschaft, sondern auch von der Anpassungsfähigkeit der mittelständischen Betriebsinhaber ab. Viele Betriebsinhaber waren von ihrem Ausbildungshintergrund her gar nicht in der Lage, den steigenden kaufmännischen Anforderungen zu genügen. Dieses ausbildungsbedingte Unvermögen ging freilich häufig Hand in Hand mit einer auf die Abwehr kapitalistisch-marktwirtschaftlicher Verhaltenszumutungen gerichteten Erwerbsmentalität.

An dieser Stelle sei auf ein Grundproblem unserer Untersuchungsergebnisse hingewiesen. Gemeint ist die mangelnde Berücksichtigung des Stadt-Land-Unterschiedes, 
zumal bei der Analyse sozialer Zusammenhänge. Daß diese regionale Differenzierung nicht stärker in den Blick geraten ist, hängt mit der Beschaffenheit der ausgewerteten Daten zusammen. Diese wurden nun einmal vorwiegend in Klein- und Großstädten erhoben. Die wenigen verfügbaren Quellen zum ländlichen Handwerk und Einzelhandel deuten darauf hin, daß sich die zwischen dörflichem und städtischem Mittelstand bestehenden Unterschiede in bezug auf Milieu, Mentalität und Lebensbedingungen nur langsam abschliffen. Sieht man vom Niedergang der traditionellen Landhandwerke ab, so hielt sich auch in ökonomischer Hinsicht der Strukturwandel der Mittelstandsbetriebe auf dem flachen Land in Grenzen.

Inwieweit ist die Dynamik der sozialökonomischen Basisprozesse auf den Einfluß staatlicher und verbandlicher Eingriffe zurückzuführen? Der zu Beginn des Untersuchungszeitraums extrem hohe Einkommensteuersatz erschwerte die innerbetriebliche Kapitalakkumulation und stellte daher eine Belastung für die kleinbetriebliche Entwicklung dar. Problematisch war zudem, daß der Einkommensteuertarif den Körperschaftsteuersatz deutlich überstieg. Der hieraus resultierende Wettbewerbsnachteil gegenüber körperschaftsteuerpflichtigen Unternehmen wurde durch einkommensteuerrechtliche Vergünstigungen in Form von Sonderabschreibungen und Freigrenzen nur teilweise ausgeglichen. $\mathrm{Zu}$ berücksichtigen ist auch, daß ein beträchtlicher Teil der mittelständischen Betriebsinhaber weder über die notwendigen Kenntnisse noch über die formalen Voraussetzungen (ordnungsgemäße Buchführung) verfügte, um die Abschreibungsmöglichkeiten in Anspruch zu nehmen. Dies ist auch der Grund, warum die sukzessive Abschaffung dieser einkommensteuerrechtlichen Anreize zur Eigenfinanzierung von den Mittelständlern kaum beklagt wurde. Die Anfang der 50er Jahre einsetzende Senkung des Einkommensteuertarifs führte zu einer nennenswerten Entlastung des gewerblichen Mittelstandes. Positiv war auch, daß sich im Zuge dieser Entwicklung der Unterschied zum Körperschaftsteuertarif verringerte. Darüber hinaus kamen insbesondere die Ausdehnung und Erhöhung der Freibeträge, die Beseitigung des Notopfers Berlin, die Einführung des Ehegattensplittings und die Korrektur des Tarifverlaufs der Kapitalbildung der Mittelstandsbetriebe zugute. Auch die Heraufsetzung der Gewerbeertragsteuer-Freigrenzen trug - wenn auch in geringerem Maße als die einkommensteuerrechtlichen Veränderungen - dazu bei, daß sich die Abgabenlast der mittelständischen Unternehmen verminderte. Nach der Gewerbesteuerreform von 1961 waren ein Drittel bis zur Hälfte der Handwerker und Einzelhändler von der Gewerbeertragsteuer gänzlich befreit. Durch das im Untersuchungszeitraum bestehende System der AllphasenBrutto-Umsatzsteuer wurden die mittelständischen Betriebe gegenüber mehrstufigen Großunternehmen benachteiligt. Die vorübergehende Erhebung von Zusatzumsatzsteuern änderte hieran wenig. Der Umsatzsteuersatz stieg im Untersuchungszeitraum an. Hierin ist ein wesentlicher Unterschied zur Entwicklung auf dem Gebiet des Einkommensteuer- und Gewerbesteuerrechts zu sehen. Der 1956 eingeführte Umsatzsteuer-Freibetrag, der 1961 deutlich erhöht wurde, führte zu einer relativen Entlastung eines Großteils der mittelständischen Unternehmen. Die konzentrationsfördernde Wirkung des Umsatzsteuersystems, die durch die Wiedereinführung der umsatzsteuerlichen Organschaft verstärkt wurde, blieb jedoch im Grundsatz weiter bestehen.

Die Anfang der 50er Jahre herrschende Kreditknappheit stellte den gewerblichen Mittelstand vor erhebliche Finanzierungsprobleme. Die Kleingewerbetreibenden taten 
sich schwer, die von den Kreditinstituten geforderten dinglichen Sicherheiten zu erbringen. Zu einer ausreichenden Kreditversorgung kam es erst Ende der 50er Jahre. Dies ist in erster Linie auf die Entspannung des privaten Kreditmarktes zurückzuführen. Gemessen an dem Kreditvolumen, das die Banken den selbständigen Mittelständlern zur Verfügung stellten, nahm sich der Umfang der staatlichen Kreditprogramme bescheiden aus. Diese hatten mithin keinen entscheidenden Einfluß auf die ökonomische Entwicklung der Klein- und Mittelbetriebe. Grundsätzlich ist zu betonen, daß die öffentlichen Darlehen den lastenausgleichsberechtigten Selbständigen in Handwerk und Einzelhandel (Vertriebene, Flüchtlinge, Kriegssachgeschädigte) in weit höherem Maße zugute kamen als den einheimischen Betriebsinhabern. In der zweiten und dritten Legislaturperiode gewann der Lenkungsaspekt bei der staatlichen Kreditförderung an Bedeutung. Als förderungswürdig galten vor allem Rationalisierungsmaßnahmen. Der Umfang dieser Programme war begrenzt. Sie erzielten keine Breitenwirkung. Dies läßt sich auch in bezug auf die gemeinschaftliche Übernahme von Kreditbürgschaften durch Bund, Länder und Verbände feststellen. Die Kreditgarantiegemeinschaften wurden erst Mitte der 50er Jahre errichtet, also zu einem Zeitpunkt, als die Talsohle in der Kreditversorgung bereits durchschritten war. Die Konditionen, zu denen Kreditbürgschaften sowie Rationalisierungskredite vergeben wurden, waren nicht sonderlich günstig. Hierin ist ein wichtiger Grund zu sehen, warum die Resonanz auf diese Maßnahmen in mittelständischen Kreisen begrenzt blieb. Abschreckend wirkten auch die umständlichen Antragsverfahren sowie die hiermit verbundene Offenlegung der Geschäftsunterlagen. Auch wenn grundsätzlich davon auszugehen ist, daß bis in die zweite Hälfte der 50 er Jahre hinein die Nachfrage mittelständischer Betriebe nach Krediten das Angebot überstieg, ist zu betonen, daß ein beträchtlicher Teil der Inhaber kleinerer Betriebe nach wie vor mentalitäts- und ausbildungsbedingte Vorbehalte gegenüber der Fremdfinanzierung hatte. Dies macht verständlich, warum Inhaber größerer Handwerks- und Einzelhandelsbetriebe unter den Empfängern staatlicher Kredithilfen und Bürgschaften überrepräsentiert waren.

Die staatlich-verbandliche Gewerbeförderung befand sich im Untersuchungszeitraum im Aufwind. In der ersten Hälfte der 50er Jahre waren die von Bund und Ländern bereitgestellten Gewerbeförderungsmittel allerdings noch vergleichsweise gering. Vernachlässigt wurde insbesondere die Förderung des mittelständischen Einzelhandels. Dies begann sich Mitte der 50er Jahre zu ändern. Die Verwendung der für Gewerbeförderungsmaßnahmen vorgesehenen Gelder erfolgte im Einvernehmen mit den Mittelstandsverbänden, die sich in der zweiten Hälfte der 50er Jahre verstärkt diesem Aufgabengebiet zuwandten. Subventioniert wurden in erster Linie der Ausbau der Fachschulen und der Mittelstandsforschung sowie die Weiterbildungsveranstaltungen für Betriebsinhaber und das mittelständische Betriebsberatungswesen. Ging es zunächst vor allem um die Vermittlung technischer Kenntnisse, so rückte in der zweiten Hälfte der 50er Jahre die betriebswirtschaftliche Schulung immer mehr in den Vordergrund. Eine Breitenwirkung wurde am ehesten bei den Vortragsveranstaltungen erzielt, die mithin als wichtige Hilfestellung bei der innerbetrieblichen Rationalisierung anzusehen sind. Lehrgänge und Arbeitsgemeinschaften waren dagegen wesentlich schlechter besucht. In den Genuß einer individuellen Betriebsberatung, die die effizienteste Form der Gewerbeförderung darstellte, kam nur ein verhältnismäßig kleiner Teil der Betriebsinhaber. Gewerbefördernde Impulse gingen auch von den örtlichen Einzelhandelsverbän- 
den und Innungen aus. Diese lokalen Fachorganisationen entwickelten sich im Untersuchungszeitraum zunehmend zu Modernisierungsagenturen. Noch stärker ausgeprägt war dieser Trend bei den genossenschaftlichen Großzusammenschlüssen des Einzelhandels. Wie bereits erwähnt, war deren Einfluß auf die Modernisierung ihrer Mitgliedsbetriebe beträchtlich. Unter dem Strich wird man festhalten können, daß die staatlich-verbandliche Gewerbeförderung zwar die innerbetriebliche Rationalisierung der Handwerks- und Einzelhandelsbetriebe vorantrieb, diese aber nicht ursächlich bedingte. Die entscheidende Triebkraft für die insbesondere in der zweiten Hälfte der 50er Jahre rasch voranschreitenden Rationalisierungsprozesse bildeten die Arbeitsmarktentwicklung - erinnert sei an den Fachkräftemangel und den daraus resultierenden Anstieg der Personalkosten -, der Wandel der Nachfragebedingungen sowie die technische Entwicklung, die die Anwendung neuer, kostengünstiger Produktions- und Absatzverfahren begünstigte.

Die mittelständischen Berufsordnungen entfalteten unterschiedliche Wirkungen. Indem sie die selbständige Berufsausübung an ein bestimmtes Ausbildungsniveau bzw. an eine entsprechende Befähigung knüpften, stellten sie den professionellen Charakter der Mittelstandsberufe sicher. Hierdurch wurden das Sozialprestige und das Zusammengehörigkeitsgefühl der Berufsangehörigen zweifellos erhöht. Das gilt für das Handwerk in weit höherem Maße als für den Einzelhandel, wo der Zugang zum Beruf in allgemeinen lediglich von einer Sach-statt einer Fachkundeprüfung abhing und der Stellenwert der Ausbildung wesentlich geringer war. Die ökonomischen Effekte der in den Berufsordnungen verankerten Marktzugangsregulierungen sind nicht zu überschätzen. Gewiß, ein Teil der potentiellen Anbieter wurde durch die Prüfungsanforderungen abgeschreckt bzw. durch das Nichtbestehen der Meisterprüfung davon abgehalten, sich selbständig zu machen. Die Handwerksordnung und die Sachkundeprüfung wirkten sich also tendenziell marktschließend aus. Wie jedoch unsere Analyse gezeigt hat, hing die Entwicklung des Betriebsbestandes und der Wettbewerbsintensität in weit höherem Maße von den Arbeitsmarktbedingungen, dem Anstieg der Betriebsgröße, den steigenden Kapitalanforderungen sowie den mit der Marktsituation zusammenhängenden Gewinnchancen ab als von den Zugangsbarrieren der Berufsordnungen. Einen durchaus nennenswerten Einfluß übten dagegen die Zugangsbeschränkungen auf das Ausbildungsniveau der Selbständigen aus. Das gilt insbesondere für das Handwerk, wo unter den Bedingungen der Gewerbefreiheit die Quote der Meisterprüfungen signifikant zurückging. Inwieweit hieraus tatsächlich ein Qualitätsverlust handwerklicher Produkte und Dienstleistungen resultierte, ist schwer zu beurteilen. Ein negativer wirtschaftlicher Effekt der Handwerksordnung ergab sich aus dem Umstand, daß sich die Zugangsbarrieren auf die einzelnen Handwerkszweige bezogen. Hierdurch wurde die Kombination verschiedener handwerklicher Tätigkeiten auf betrieblicher Ebene erschwert. Durch die 1965 erlassene Novelle zur Handwerksordnung wurde dieses Problem entschärft.

Was die Wirkungen des Gesetzes gegen Wettbewerbsbeschränkungen betrifft, so ist zunächst festzustellen, daß die Großunternehmen von den hierin verankerten weitreichenden Ausnahmen vom Kartellverbot ungleich mehr profitierten als die mittelständischen Betriebe. Wie sich bald nach Inkrafttreten des Gesetzes herausstellte, war das GWB zudem nicht geeignet, die Unternehmenskonzentration aufzuhalten. Dies dürfte inbesondere die Entwicklung jener Mittelstandsbetriebe beeinträchtigt haben, die in direkter Konkurrenz mit den begünstigten Großbetrieben standen. Wie nachgewiesen 
werden konnte, waren direkte Konkurrenzbeziehungen zwischen Handwerk und Industrie eher die Ausnahme als die Regel. Man wird daher davon ausgehen können, daß die Wachstumschancen, die sich für den Mittelstand aus der industriellen Expansion ergaben, die negativen Konkurrenzwirkungen überwogen.

Vereinigungen kleiner und mittlerer Unternehmen wurde im GWB das Recht eingeräumt, unter bestimmten Bedingungen Preis- und Kalkulationsempfehlungen auszusprechen. Inwieweit sich infolge dieser mittelstandsspezifischen Ausnahmeregelung die Wettbewerbsposition der mittelständischen Betriebe verbesserte oder ob sich die Mittelstandsempfehlungen primär dahingehend auswirkten, das traditionelle Preisbildungsverhalten der Mittelständler zu festigen, so daß sich die modernisierenden Wirkungen des Preiswettbewerbs nicht voll entfalteten, muß offenbleiben. Lediglich in bezug auf die genossenschaftlichen Großzusammenschlüsse des mittelständischen Einzelhandels kann festgestellt werden, daß die Möglichkeit, eine koordinierte Preispolitik zu betreiben, die Leistungs- und damit auch die Konkurrenzfähigkeit der Mitgliedsbetriebe erhöhte. Die Wiederzulassung der Preisbindung der zweiten Hand zeitigte in bezug auf die ökonomische Entwicklung des mittelständischen Einzelhandels ebenfalls ambivalente Wirkungen: Zum einen wurde hierdurch der Preiswettbewerb auf der Handelsstufe ausgeschaltet und das traditionelle Spannendenken gefördert, was zunächst vor allem den Kleinbetrieben zugute kam. Zum anderen stellte die vertikale Preisbindung eine wesentliche Voraussetzung für die massenhafte Distribution von Markenartikeln dar, was wiederum die Rationalisierung der Absatztechniken vorantrieb. Die Preisbindung der zweiten Hand ist übrigens ein gutes Beispiel dafür, daß die wirtschaftliche Modernisierung nicht immer im Widerspruch zu traditionellen Verhaltensmustern stand.

Ob die im Wirtschaftsstrafgesetz enthaltenen Strafandrohungen ein effizientes Mittel darstellten, um die Inhaber von Klein- und Mittelbetrieben zu einer wettbewerbsgerechten Preisbildung und damit zu einer Senkung des Preisniveaus zu veranlassen, darf bezweifelt werden. Die große Zahl der von den Preisüberwachungsbehörden nachgewiesenen Preisabsprachen deutet darauf hin, daß die von der Preistreibereibestimmung ausgehende Abschreckungswirkung verhältnismäßig gering war. Ein beträchtlicher Teil der Mittelständler scheint an traditionellen Preisbildungsmethoden festgehalten und sich somit über die gesetzlichen Bestimmungen hinweggesetzt zu haben.

Neben dem Kartellgesetz gab es eine Reihe weiterer wettbewerbspolitischer Eingriffe, die den gewerblichen Mittelstand betrafen. Für die Wettbewerbsbedingungen im handwerklichen Bereich war vor allem das 1957 verabschiedete Gesetz zur Bekämpfung der Schwarzarbeit relevant. Das Handwerk erhoffte sich hiervon einen wirksamen Schutz vor „illegitimer" Konkurrenz. Diese Erwartungshaltung wurde indes nur ansatzweise erfüllt, da das Gesetz nur auf schwere Fälle der Schwarzarbeit abgestellt war. Darüber hinaus ließen die Tatbestandsmerkmale die nötige Präzision vermissen. Effizienzmindernd wirkte sich zudem der Umstand aus, daß die entsprechenden Verstöße in der Praxis nur schwer nachweisbar waren. Die 1954 erfolgte Reform des Genossenschafts- und Rabattgesetzes hielt ebenfalls nicht das, was sich der mittelständische Einzelhandel von ihr versprach. Die Warenrückvergütung, die einen entscheidenden Anreiz für die Konsumvereinsmitgliedschaft darstellte, wurde zwar auf $3 \%$ begrenzt, die Konsumgenossenschaften gingen jedoch bald dazu über, eine Niedrigpreispolitik zu betreiben. Infolge dieser preispolitischen Offensive dürfte sich der von den Konsumgenossenschaften ausgehende Wettbewerbsdruck auf den mittelständischen Nahrungsmittel- 
einzelhandel wohl kaum verringert haben. Daß der Marktanteil der Konsumgenossenschaften in der Folgezeit dennoch zurückging, hing weniger mit dem Gesetzgebungsakt von 1954 als mit der Expansion der Einkaufsgenossenschaften und freiwilligen Ketten sowie der Entstehung neuer Großbetriebsformen zusammen.

Vom Ladenschlußgesetz gingen insofern modernisierende Wirkungen aus, als die Verkürzung der Öffnungszeiten jene Betriebe begünstigte, die sich zeitsparender Absatztechniken bedienten. Darüber hinaus hatte die Vorverlegung des Ladenschlusses von 19 auf 18.30 Uhr einen betriebsgrößenstrukturellen Effekt: Da sich die Kleinbetriebe im Gegensatz zu Einzelhandelsgroßbetrieben in den seltensten Fällen Schichtarbeit leisten konnten, profitierten sie von der Verringerung des Unterschieds zwischen der Ladenöffnungszeit und der Arbeitszeit der Angestellten - allerdings nur unter der Voraussetzung, daß sie sich rationalisierungswillig zeigten. Aus sozialgeschichtlichem Blickwinkel ist bedeutsam, daß die Abschaffung des „langen Samstags“ zu einem Freizeitzuwachs für die selbständigen Einzelhändler führte.

Wettbewerbspolitik wurde nicht nur vom Staat, sondern auch von den Verbänden betrieben. Hervorzuheben ist hier insbesondere das zwischen der HDE und der BAG im Sommer 1952 geschlossene Expansionsstoppabkommen, in dem sich der Verband der Einzelhandelsgroßbetriebe verpflichtete, seinen Mitgliedsunternehmen zu empfehlen, für die Dauer von 2 Jahren keine neuen Verkaufsstellen zu errichten. Abgesehen davon, daß keineswegs alle Mittel- und Großbetriebe diesen Aufruf beherzigten, änderte der vorübergehende Expansionsverzicht nichts daran, daß sich das Wachstum der Großbetriebsformen im Untersuchungszeitraum fortsetzte. Ende der 50er Jahre richtete die Einzelhandelsführung erneut Maßhalteappelle an die nichtmittelständischen Einzelhandelsunternehmen. Die Bemühungen der HDE, den Strukturwandel im Einzelhandel aufzuhalten, erwiesen sich jedoch als wenig erfolgreich.

Von der Gesetzgebung auf dem Gebiet des Arbeitsrechts war nur ein kleiner Teil der Handwerks- und Einzelhandelsbetriebe direkt betroffen. Das gilt insbesondere für das Kündigungsschutzgesetz von 1951 und das Betriebsverfassungsgesetz von 1952. Die Mehrzahl der mittelständischen Unternehmen fiel nicht in den Geltungsbereich dieser Gesetze. $\mathrm{Zu}$ einer Verrechtlichung der Arbeitsbeziehungen kam es lediglich in etwas größeren Mittelstandsbetrieben. Den Inhabern dieser Betriebe war es fortan nicht mehr möglich, Arbeitskräfte kurzfristig zu entlassen. Inwieweit dieser Flexibilitätsverlust die betriebliche Leistungsfähigkeit beeinträchtigte, entzieht sich unserer Kenntnis. Man wird davon ausgehen können, daß sich in jenen Betrieben, die die Bestimmungen des Betriebsverfassungsgesetzes nicht umsetzten, die schlechtere arbeitsrechtliche Lage auf die Rekrutierung von Arbeitskräften negativ auswirkte. In der frühen Bundesrepublik spielte dieser Gesichtspunkt angesichts der hohen Arbeitslosigkeit eine untergeordnete Rolle. Im Zuge der zunehmenden Arbeitskräfteknappheit änderte sich dies freilich. Die Wirkungsgeschichte des 1960 verabschiedeten Jugendarbeitsschutzgesetzes fällt nicht mehr in unseren Untersuchungszeitraum und ist daher nicht näher analysiert worden. Aufschlußreich ist, daß ein beträchtlicher Teil der Handwerks- und Einzelhandelsbetriebe keine Lehrlinge beschäftigte und somit weder von den Jugendarbeitsschutzbestimmungen noch von der arbeitsmarktbedingten Erhöhung der Lehrlingsvergütungen unmittelbar betroffen war. Grundsätzlich wird man allerdings davon ausgehen können, daß die Bereitschaft, Jugendliche auszubilden und zu beschäftigen, mit der Verteuerung der Lehrlingsarbeit abnahm. 
Durch das Handwerkerversorgungsgesetz von 1938 und - in seiner Nachfolge - das Handwerkerversicherungsgesetz von 1960 verbesserte sich die Alterssicherung der selbständigen Handwerker. Allerdings ist zu betonen, daß die Handwerkerrenten auch nach der gesetzlichen Neuregelung von 1960 in den meisten Fällen nur einen Zuschuß zum Lebensunterhalt darstellten. Sozialgeschichtlich bedeutsam ist auch die durch die Einbeziehung der Handwerker in die Angestellten- bzw. Invalidenversicherung bedingte versicherungsrechtliche Nivellierung des Arbeitnehmer-Arbeitgeber-Unterschiedes. Dies dürfte zur Unterminierung des berufsständischen Sonderstatus beigetragen haben.

Die von den Mittelstandsverbänden immer wieder geäußerte Behauptung, die gesetzlichen Sozialabgaben hätten den gewerblichen Mittelstand wesentlich stärker belastet als die Großbetriebe, trifft in dieser allgemeinen Form nicht zu. Die institutionelle Lohn- bzw. Gehaltsintensität war insbesondere in Kleinstbetrieben, wo neben dem Betriebsinhaber und vielleicht der Ehefrau nur wenige Lohnabhängige beschäftigt waren, vergleichsweise gering. Hinzu kommt, daß die Mehrzahl der Handwerks- und Einzelhandelsbetriebe durchaus in der Lage war, die lohnbezogenen Sozialabgaben auf die Preise zu überwälzen.

Es hat den Anschein, als habe die nichtstaatliche Sozialpolitik die sozialökonomische Entwicklung des gewerblichen Mittelstandes in mancherlei Hinsicht stärker bestimmt als die sozialpolitische Gesetzgebung. So ist in dem Anstieg der freiwilligen betrieblichen Sozialleistungen in Großbetrieben einer der wesentlichen Gründe zu sehen, warum seit Mitte der 50er Jahre immer mehr Arbeitnehmer aus dem Handwerk abwanderten bzw. sich von vornherein für eine berufliche Laufbahn in Großunternehmen entschieden. Diese Entwicklung traf die mittelständischen Unternehmen vor dem Hintergrund der zunehmenden Arbeitskräfteknappheit besonders hart. Sie hatte allerdings auch eine positive Seite: Die wachsenden Probleme bei der Arbeitskräfterekrutierung förderten die Abkehr von personalintensiven Produktions- und Absatzverfahren und trugen somit zur Modernisierung von Handwerk und Einzelhandel bei. Ein ähnlicher Effekt resultierte aus der 1956 einsetzenden Arbeitszeitverkürzung und dem gleichzeitigen Anstieg der Löhne und Gehälter. Die mittelständischen Betriebe übernahmen zwar bald die zwischen Industrie und Gewerkschaften vereinbarten Arbeitszeitarrangements; dies hatte jedoch zur Folge, daß sich die Arbeitskraft verteuerte, was wiederum Rationalisierungsmaßnahmen sinnvoll erscheinen ließ. Des weiteren bewirkte die Arbeitszeitverkürzung, daß sich in bezug auf das Freizeitbudget die Schere zwischen mittelständischen Selbständigen und Arbeitnehmern allmählich zu öffnen begann, was die Attraktivität der selbständigen Berufsausübung nicht eben erhöht haben dürfte.

Betrachtet man die untersuchten mittelstandsrelevanten Interventionen im Überblick, so ist zunächst festzustellen, daß die rechtlich bedingten Wettbewerbsnachteile der mittelständischen Betriebe gegenüber den Großunternehmen in der zweiten und dritten Legislaturperiode sukzessive abgebaut wurden, zumal auf dem Gebiet der Steuer- und Kreditpolitik. Sieht man von den tendenziell erhaltungsinterventionistischen Wirkungen der mittelständischen Berufsordnungen sowie einiger wettbewerbspolitischer Gesetze und Maßnahmen ab, so trugen die staatlichen und verbandlichen Eingriffe - soweit sich deren Wirkungen überhaupt bestimmen lassen - im allgemeinen dazu bei, daß sich der gewerbliche Mittelstand an die neuen ökonomischen Bedingungen anpaßte. 
Grundsätzlich ist allerdings zu betonen, daß die Wirkung der politischen Steuerungsversuche eng begrenzt war. Der sozialökonomische Strukturwandel wurde in erster Linie durch die sich verändernden ökonomischen und sozialen Rahmenbedingungen vorangetrieben und nicht durch mittelstandsbezogene Interventionen des Staates oder der Verbände. Letztere hatten vielmehr den Charakter flankierender Maßnahmen. Als entscheidende Triebkräfte des Wandels sind die kollektive Erhöhung des Lebensstandards und die daraus resultierenden quantitativen und qualitativen Nachfrageverschiebungen, die siedlungsstrukturellen Veränderungen, die zunehmende Technisierung von Wirtschaft und Gesellschaft, die Entwicklung des Arbeitsmarktes sowie die industrielle Expansion anzusehen. Die genannten Prozesse, die eng miteinander verflochten waren, setzten die selbständigen Handwerker und Kleinhändler unter einen gewaltigen Anpassungsdruck. Dieser Gesamtbefund ist in zweifacher Hinsicht zu präzisieren: Ohne die staatlicherseits geschaffenen ordnungspolitischen Voraussetzungen hätte sich die „Eigengesetzlichkeit" der wirtschaftlichen Dynamik wohl kaum in dem dargestellten $\mathrm{Maße}$ entfalten können. Des weiteren ist zu berücksichtigen, daß im Rahmen der vorliegenden Untersuchung nur jene Maßnahmen und Gesetze untersucht wurden, die den gewerblichen Mittelstand direkt betrafen. Die Frage, inwieweit die allgemeinen Eingriffe in den gesamtwirtschaftlichen Prozeß sowie die sektorspezifischen Regulierungen in anderen Wirtschaftsbereichen die wirtschaftliche Lage der Klein- und Mittelbetriebe in Handwerk und Einzelhandel beeinflußten, muß mithin offenbleiben.

Fragt man nach den strukturellen Bedingungen für die politischen Durchsetzungschancen der Mittelstandsinteressen, so kommt man nicht umhin, das berufsgruppeninterne Interessenvermittlungssystem in den Blick zu nehmen. Der nach dem Krieg erfolgende Wiederaufbau des mittelständischen Verbandswesens war von dem Konflikt zwischen fachlichem und überfachlichem Organisationszweig geprägt. Rangen im Handwerk die Kammern und die Fachverbände um die innerorganisatorische Vorherrschaft, so waren es im Einzelhandel die Regionalverbände und die Branchenvertretungen. Die sowohl machtpolitisch als auch konzeptionell bedingten Spannungen waren zwischenzeitlich so stark, daß ein Auseinanderbrechen der Mittelstandsverbände drohte. Wäre es tatsächlich zu einer organisatorischen Fragmentierung gekommen, so hätten sich die Einflußchancen des gewerblichen Mittelstandes auf die Entscheidungsbildung im staatlichen Bereich zweifellos gemindert. Im Einzelhandel konnte das traditionelle Organisationsproblem, das in der fachlich-organisatorischen Zersplitterung bestand, durch die institutionelle Aufwertung des überfachlichen Organisationszweiges weitgehend überwunden werden. Der innerverbandliche Einfluß der Fachverbände wurde zugunsten der Regionalverbände zurückgedrängt. Als problematisch erwies sich dagegen die Integration der Großbetriebe in das vorwiegend mittelständisch geprägte Verbandswesen. So konnte nicht verhindert werden, daß sich eine separate Interessenvertretung der Mittelund Großbetriebe bildete. Die BAG war zwar Mitglied des Gesamtverbandes, hatte dort jedoch nur eine Außenseiterstellung inne, da sie jederzeit von den Mittelstandsvertretern, die in den Verbandsgremien in der Überzahl waren, majorisiert werden konnte. Die Einzelhandelsführung um Hans Schmitz, dem ersten Präsidenten der Hauptgemeinschaft, bemühte sich um einen Ausgleich zwischen groß- und kleinbetrieblichen Interessen. Ähnlich wie in der Weimarer Republik, lief sie dabei jedoch permanent $\mathrm{Ge}-$ fahr, zwischen der mittelständischen Basis auf der einen und den Großbetrieben auf der 
anderen Seite zerrieben zu werden. In der Handwerksorganisation spielte der betriebsgrößenstrukturelle Konflikt eine wesentlich geringere Rolle. Das organisatorische Hauptproblem bestand darin, eine Synthese zwischen den Fachverbänden, die sich als reine Unternehmervertretungen verstanden, und den berufsständisch ausgerichteten Selbstverwaltungsorganen zu finden. Letztere hatten de iure und ihrem Selbstverständnis zufolge die Aufgabe, die Belange der selbständigen und der abhängig beschäftigten Handwerker zu vertreten, wenngleich sie de facto die Interessen der Betriebsinhaber privilegierten. Der ZDH stand als handwerkliche Spitzenvertretung zwischen diesen beiden Blöcken. Nachdem die Rivalitäten zwischen Kammern und Fachverbänden abgeklungen waren, verbesserten sich die Voraussetzungen für eine einheitliche Willensbildung und damit auch für eine erfolgreiche Interessenvertretungspolitik des $\mathrm{ZDH}$ gegenüber Legislative und Exekutive. Einen ähnlichen Effekt hatte auch die Tatsache, daß die Handwerkerbündebewegung, die in der Weimarer Republik die Autorität des handwerklichen Spitzenverbandes untergraben hatte, nach dem Krieg nicht wieder auflebte. In der Handwerksordnung von 1953 wurde der öffentlich-rechtliche Status der Kammern und Innungen, der in der amerikanischen Zone vorübergehend beseitigt worden war, gesetzlich verankert. Damit war im Kern die Organisationsstruktur der Vorkriegszeit wieder hergestellt. Ein Novum stellte die Drittelparität der Gesellen in den Handwerkskammern dar. Der Einfluß der handwerklichen Arbeitnehmer auf die Entscheidungsbildung der Selbstverwaltungsorgane erhöhte sich durch die Einführung der überbetrieblichen Mitbestimmung jedoch nur unwesentlich.

In der unmittelbaren Nachkriegszeit wurde die Bedeutung des gewerblichen Mittelstandes für die zukünftige wirtschaftliche und gesellschaftliche Entwicklung von nahezu allen maßgeblichen politischen Kräften sehr hoch eingestuft. Die ökonomische Existenzberechtigung der Klein- und Mittelbetriebe wurde auch von der SPD anerkannt. Vor dem Hintergrund der Weimarer Erfahrungen hielt es Schumacher für unabdingbar, daß sich die Partei gegenüber den kleinen Selbständigen öffnete. Hierbei spielten wahltaktische Motive ebenso eine Rolle wie die Überzeugung, daß das antidemokratische Potential der mittelständischen Gewerbetreibenden entschärft werden müsse. Adenauer sah demgegenüber in der Existenz einer breiten Selbständigenschicht eine wesentliche Voraussetzung für eine erfolgreiche Demokratisierung. Darüber hinaus stellte die Selbständigkeit für die Christdemokraten einen Wert an sich dar. Der Mittelstand wurde insbesondere von den Vertretern der katholischen Soziallehre als Trägerschicht eines christlichen Sozialmodells angesehen. Die besondere Wertschätzung der Kleinund Mittelbetriebe hing zudem mit den in den ersten Nachkriegsjahren weitverbreiteten Vorbehalten gegenüber industriellen Großbetrieben zusammen. Auch die Neoliberalen bekannten sich zu diesem Zeitpunkt zur positiven Utopie einer dezentralisierten Wirtschaftsstruktur. Dies erklärt, warum trotz der heftigen wirtschaftspolitischen Flügelkämpfe, die Ende der 40er Jahre innerhalb der Union tobten, die Mittelstandsförderung als programmatisches Ziel nicht in Frage gestellt wurde. Sieht man von den frühen Parteiprogrammen der CSU ab, so ist festzustellen, daß keine der großen Parteien für einen bedingungslosen Schutz des gewerblichen Mittelstandes eintrat. Am wenigsten protektionistisch war die FDP, was die Zusammenarbeit mit den Mittelstandsverbänden nicht eben erleichterte.

Die mittelstandsfreundliche Programmatik der Parteien stand in der frühen Bundesrepublik in krassem Widerspruch zur politischen Praxis. Angesichts der krisenhaften 
Zuspitzung der wirtschaftlichen und sozialen Lage erschien der Bundesregierung die Berücksichtigung mittelstandsspezifischer Interessen nicht vordringlich. Die politischen Entscheidungsträger richteten ihre Politik am Primat der industriellen Entwicklung aus. Dies wurde überdeutlich, als es nach Ausbruch des Korea-Krieges im Sommer 1950 zu Engpässen im Bereich der Eisen-, Stahl- und Kohleversorgung kam. Die Großwirtschaft hatte bei der Zuteilung von Rohstoffen im Zweifelsfall Vorrang vor mittelständischen Betrieben. Hiervon war das Handwerk stärker betroffen als der Kleinhandel. Die Privilegierung der industriellen Großbetriebe stand in Kontintuität zur Bewirtschaftungspraxis der unmittelbaren Nachkriegszeit. Zu einer Benachteiligung der Kleinund Mittelbetriebe kam es auch auf dem Gebiet der Kreditpolitik. Als prioritätsberechtigte Gruppen galten hier vor allem die Grundstoffindustrie, der Export und die Landwirtschaft. Auch im sozialpolitischen Bereich war den Forderungen von Handwerk und Einzelhandel nur wenig Erfolg beschieden. Dies ist nicht zuletzt auf die Struktur des politischen Interessenvermittlungssystems Anfang der 50er Jahre zurückzuführen. Die bis zum Frühjahr 1952 andauernde enge Kooperation zwischen Gewerkschaften, Industrie und Staat, deren Entstehung mit den außenpolitischen Zielen Adenauers, der labilen wirtschaftlichen Lage sowie der in der ersten Legislaturperiode starken Stellung des arbeitnehmerfreundlichen Flügels in der Union zusammenhing, beeinträchtigte die politischen Durchsetzungschancen der Mittelstandsverbände. Der ZDH, der sich in die Defensive gedrängt sah, versuchte seine Einflußchancen durch die politische Mobilisierung seiner Klientel zu erhöhen. Anknüpfend an die ideologischen Feindbilder des gewerblichen Mittelstands in der Weimarer Republik, stilisierte der handwerkliche Spitzenverband im Sommer 1951 die Abschaffung der „Warenhaussteuer" und das Kündigungsschutzgesetz zu zentralen Fragen der Mittelstandspolitik, obwohl die tatsächlichen Auswirkungen dieser staatlichen Eingriffe auf die wirtschaftliche Situation der Klein- und Mittelbetriebe vergleichsweise gering waren. Die Reideologisierung der Verbandspolitik stieß bei der Basis auf positive Resonanz. Dabei ist zu berücksichtigen, daß sich der Wettbewerbsdruck Anfang der 50er Jahre im kleinbetrieblichen Bereich deutlich erhöhte, was sich insbesondere im Rückgang der Alleinmeisterbetriebe manifestierte. Angesichts der realen ökonomischen Bedrohung waren Schuldzuweisungen an die Adresse des Staates und der Sozialpartner nach dem Muster der traditionellen Mittelstandsideologie durchaus populär.

Die Einzelhandelsführung, die sich als Gesamtvertretung der Klein- und Großbetriebe verstand, distanzierte sich von dem politischen Konfrontationskurs des ZDH, da sie im Falle einer zunehmenden Radikalisierung der mittelständischen Basis ein Auseinanderbrechen ihrer Organisation befürchtete. Sie beteiligte sich auch nicht an der im September 1951 erfolgenden Gründung des Deutschen Mittelstandsblocks. Das erklärte Ziel dieses organisatorischen Zusammenschlusses war es, den Mittelstand zum "dritten Sozialpartner" zu machen. Der politische Einfluß der Gewerkschaften und der Großindustrie sollte zurückgedrängt und der Staat zu einer stärkeren Berücksichtigung mittelstandsspezifischer Interessen veranlaßt werden. Träger des Mittelstandsblocks waren zunächst die Spitzenverbände des Handwerks, der Landwirtschaft sowie des Hausund Grundbesitzes. Die Bundesregierung, deren Aufmerksamkeit in der Frühphase der Bundesrepublik vor allem dem Radikalisierungspotential der Vertriebenen galt, reagierte auf die Bildung dieser mittelständischen Sammlungsbewegung ebenso überrascht wie beunruhigt. Die Regierungsparteien vermuteten in dem DMB eine Neuauflage der 
Weimarer Mittelstandspartei und betrachteten ihn daher als Bedrohung ihrer Wählerbasis. Die DMB-Führung, in der der ZDH den Ton angab, bestritt zwar, auf parlamentarischer Ebene mit den etablierten Parteien konkurrieren zu wollen, schloß aber auch nicht aus, daß es zu einer derartigen Entwicklung kommen könne, wenn die Forderungen des Mittelstandes weiterhin unberücksichtigt blieben. Angesichts dieser latenten Drohung gingen die bürgerlichen Parteien in der Folgezeit nicht nur verstärkt auf die Anliegen der Mittelstandsverbände ein, sie arbeiteten auch im Vorfeld der Landtagsund Kommunalwahlen mit dem Mittelstandsblock zusammen. Der dezidiert antisozialistisch eingestellte DMB wandelte sich vom einem potentiellen Konkurrenten im Kampf um Wählerstimmen zu einem konservativen Partner im außerparlamentarischen Bereich. Die Möglichkeit einer mittelständischen Parteibildung stand spätestens seit dem Ende der ersten Legislaturperiode nicht mehr zur Debatte. Das anfänglich so wirksame Drohpotential des DMB nahm infolgedessen ab. Daß der Mittelstandsblock nach 1953 sehr bald an Bedeutung verlor, hing überdies mit dem Unvermögen seiner Trägerverbände zusammen, sich über die politische Zielsetzung des DMB zu einigen. Die innerverbandliche Konsensbildung wurde zudem durch die immer heterogener werdende Mitgliederstruktur erschwert. Nach dem im Juli 1952 erfolgenden Beitritt des Deutschen Beamtenbundes zum DMB veränderte sich die programmatische Grundlage des mittelständischen Zusammenschlusses: War die Blockbildung anfangs auf den selbständigen Mittelstand beschränkt, so wurde sie nunmehr zum Interessenvertretungsorgan der „mittelständischen Schichten“, einschließlich des neuen Mittelstandes und der Arbeiterelite. Im Frühjahr 1953 trat schließlich auch die HDE unter dem Druck ihrer mittelständischen Basis dem DMB bei. Hierdurch wurde die Handlungsfähigkeit des DMB weiter eingeschränkt, vertraten doch Landwirtschaft und Einzelhandel in zentralen politischen Punkten unterschiedliche Interessen. Ende 1957 wurde der Mittelstandsblock schließlich wegen seiner nunmehr offenkundigen Ineffizienz aufgelöst. Kurz vorher hatten sich die Verbände des gewerblichen Mittelstandes zu einer informellen Arbeitsgemeinschaft zusammengeschlossen. Insbesondere $\mathrm{ZDH}$ und $\mathrm{HDE}$ begannen jetzt enger zusammenzuarbeiten.

In der zweiten und dritten Legislaturperiode verbesserten sich die politischen Durchsetzungschancen des gewerblichen Mittelstandes. Die Interessen der Klein- und Mittelbetriebe wurden sowohl in der Steuer- als auch in der Kredit- und Wettbewerbspolitik in zunehmenden Maße berücksichtigt. Die Gewerbeförderung machte ebenfalls Fortschritte. Auf dem Gebiet der Sozialgesetzgebung konnten sich $\mathrm{ZDH}$ und HDE dagegen nur bedingt durchsetzen. Eine bedeutende Ausnahme stellte hier freilich das Handwerkerversicherungsgesetz dar, bei dem die Handwerker gegenüber der Versichertengemeinschaft der Arbeiter bevorzugt wurden.

Welche strukturellen Veränderungen begünstigten die zunehmende Berücksichtigung der Mittelstandsforderungen? Die positive Wende in der Mittelstandspolitik ist vor dem Hintergrund des wirtschaftlichen Aufschwungs zu sehen. Erst als sich die ökonomischen Verteilungsspielräume zu öffnen begannen, erschien es der Regierungskoalition vertretbar, die Mittelstandsförderung zu forcieren. Die mittelstandspolitische Kurskorrektur hing zudem mit dem wachsenden Einfluß der Mittelstandsvertreter innerhalb der CDU/CSU-Fraktion zusammen. Dies wiederum ist nicht zuletzt auf den organisatorischen Zusammenschluß der Mittelstandsvertreter in Partei und Fraktion zurückzuführen. Der gegen Ende der ersten Legislaturperiode konstitiuierte Diskussions- 
kreis Mittelstand, der in engem Kontakt mit den Mittelstandsverbänden stand, setzte sich dezidiert für die Interessen der Klein- und Mittelbetriebe ein. Seit Mitte der 50er Jahre gelang es den Mittelstandsvertretern der Union immer häufiger, sich innerhalb der Fraktion gegenüber konkurrierenden Interessentengruppen durchzusetzen bzw. diese für ihre Ziele zu gewinnen. Dabei kam dem DKM zugute, daß der fraktionsinterne Einfluß der Arbeitnehmervertreter, die den Forderungen der Mittelstandsverbände distanziert bis ablehnend gegenüberstanden, aufgrund der sich wandelnden Mehrheitsverhältnisse in der zweiten und dritten Wahlperiode geringer war als in der ersten. Der 1953 errichtete Bundestagsausschuß für Sonderfragen des Mittelstandes, dem fast ausschließlich mittelstandsnahe Abgeordnete angehörten, trug ebenfalls dazu bei, daß im Rahmen der legislatorischen Arbeit den Belangen der Kleingewerbetreibenden zunehmend Beachtung geschenkt wurde. Zu Beginn der zweiten Legislaturperiode intensivierte auch die auf dem Weg zur Staatspartei befindliche SPD ihre Mittelstandsarbeit. Wo immer es sich mit den Grundsätzen der Partei vereinbaren ließ, setzte sie sich für die Forderungen der Mittelstandsverbände ein. Reibungspunkte ergaben sich allerdings dann, wenn - wie in der Sozialpolitik - die Interessen der kleinen Selbständigen mit denen der Arbeitnehmer im Widerspruch standen. Trotz ihres verstärkten mittelstandspolitischen Engagements gelang es der SPD im Untersuchungszeitraum weder die Betriebsinhaber in Handwerk und Einzelhandel in nennenswertem Ausmaß an sich zu binden noch die Mittelschichtenarbeit innerhalb der Partei auf ein breites organisatorisches Fundament zu stellen. Zu einer institutionellen Aufwertung des gewerblichen Mittelstandes kam es nicht nur in den Parteien, sondern auch in der Ministerialbürokratie. Um die Jahreswende 1951/52 wurden die im Bundeswirtschaftsministerium für Handwerks- und Einzelhandelsfragen zuständigen Referate zu Unterabteilungen ausgebaut. Ende der zweiten Legislaturperiode wurde darüber hinaus ein Referat für Mittelstandsfragen eingerichtet. Das Bestreben, die Probleme des gewerblichen Mittelstandes im Zusammenhang zu bearbeiten, drückte sich zudem in der 1959 erfolgenden Konstituierung eines „Interministeriellen Mittelstandsausschusses“ aus. Der Ausbau der für Mittelstandsfragen zuständigen Stellen spiegelte die zunehmende Relevanz der Mittelstandspolitik wider. Für die mittelständischen Berufsgruppen, die über ausgezeichnete Beziehungen zu den Mittelstandsreferenten des BMW verfügten, war diese Entwicklung positiv, erhöhten sich hierdurch doch ihre Einflußchancen auf den regierungsinternen Entscheidungsprozeß.

Inwieweit war die staatliche Mittelstandspolitik der 50er Jahre sozialprotektionistisch motiviert, und welchen Stellenwert nahm die Förderung von Handwerk und Kleinhandel in der Zielhierarchie der Bundesregierung ein? Mittelstandsprotektionistische Intentionen spielten insbesondere bei der Gestaltung der Handwerksordnung und des Einzelhandelsgesetzes eine zentrale Rolle. Wie bereits erwähnt, wirkte sich der Große Befähigungsnachweis tendenziell wettbewerbsbeschränkend aus. Obgleich dieser Effekt absehbar war, stimmten 1953 - mit Ausnahme der KPD - alle im Bundestag vertretenen Parteien der HwO zu. Die 1948 in der amerikanischen Zone eingeführte Gewerbefreiheit blieb Episode. Die Zustimmung zur Handwerksordnung wurde sowohl von den bürgerlichen Parteien als auch von der SPD damit begründet, daß nur durch den Großen Befähigungsnachweis ein Qualitätsverlust der Handwerksarbeit verhindert werde könne. Der freie Wettbewerb sei hierzu nicht in der Lage. Bemerkenswert ist, daß auch Erhard diesen Standpunkt teilte, obwohl er ansonsten entschieden für ge- 
werbefreiheitliche Prinzipien eintrat. Diese befürwortende Haltung ist nicht zuletzt mit dem hohen Prestige zu erklären, das das Handwerk aufgrund seiner berufsständischen Tradition genoß. Von dieser Aura konnten sich offenbar auch die Neoliberalen nicht frei machen. Darüber hinaus spielten wahltaktische Gesichtspunkte eine Rolle. Immerhin stand die Forderung nach der gesetzlichen Verankerung des Großen Befähigungsnachweises auf der handwerkspolitischen Agenda des ZDH ganz oben. Wer sich für den freien Marktzugang aussprach, lief Gefahr, den Unmut der etablierten Handwerker auf sich zu ziehen. Die Durchsetzungsfähigkeit der Handwerksinteressen in dieser Frage wurde durch zwei weitere Umstände begünstigt: Zum einen entstanden dem Staat durch die wettbewerbspolitische Absicherung des Handwerks - anders als z. B. in der Steuer- und Kreditpolitik - keine direkten Kosten, und zum anderen gab es keine einflußreichen organisierten Gegeninteressen. Die von den Zugangsbarrierren negativ Betroffenen waren in erster Linie die Verbraucher. Diese ließen sich jedoch nur begrenzt organisieren. Hinzu kommt, daß der Große Befähigungsnachweis von einem Großteil der Bevölkerung durchaus akzeptiert wurde. Die politischen Widerstände gegen die von der HDE geforderte Zugangsbeschränkung im Einzelhandel waren wesentlich größer als gegen die Handwerksordnung. Dies ist nicht zuletzt darauf zurückzuführen, daß die berufsständische Tradition im Einzelhandel vergleichsweise schwach ausgeprägt war und daher nicht als Legitimationsgrundlage für die geforderte Berufsordnung ins Feld geführt werden konnte. Außerdem stuften Regierung und Parteien die ökonomische Bedeutung des Kleinhandels deutlich tiefer ein als die des Handwerks. Eine Privilegierung dieses Wirtschaftsbereiches erschien somit nicht notwendig. Erhard stand der Beschränkung des Marktzugangs im Einzelhandel, in der er eine wettbewerbsfeindliche Maßnahme erblickte, äußerst kritisch gegenüber. Ebenso wie die SPD, die gegen das Einzelhandelsgesetz stimmte, stand der Bundeswirtschaftsminister jedoch vor dem Problem, daß er nicht rational begründen konnte, warum er den Großen Befähigungsnachweis befürwortete, den Sachkundenachweis dagegen, obwohl dessen Eingriffsintensität wesentlich geringer war, wegen seines marktinkonformen Charakters ablehnte. Nachdem das Bundesverwaltungsgericht die Verfassungsmäßigkeit des Sachkundenachweises bestätigt hatte, setzten sich die Mittelstandsvertreter in der Unionsfraktion und die Sachwalter des Einzelhandels im Bundeswirtschaftsministerium gegen Erhard durch, so daß das Einzelhandelsgesetz im Jahre 1957 verabschiedet werden konnte.

Sieht man von den mittelständischen Berufsordnungen ab, so wird man feststellen können, daß es den politisch Verantwortlichen in Regierung und Parlament im allgemeinen nicht darum ging, den Mittelstand vor Konkurrenz zu schützen. Der Gesetzgeber orientierte sich vielmehr am Leitbild der Wettbewerbsneutralität zwischen Groß- und Kleinbetrieben. Dies läßt sich an den steuerpolitischen Reformen der 50er Jahre ebenso nachweisen wie an der mittelstandsrelevanten wettbewerbspolitischen Gesetzgebung. Des weiteren ist hervorzuheben, daß die staatliche Mittelstandspolitik weniger auf die Konservierung als auf die Modernisierung der mittelständischen Wirtschaft zielte. In diesem Zusammenhang ist vor allem auf die Kreditpolitik und die Gewerbeförderung $\mathrm{zu}$ verweisen.

Einem erheblichem Teil der für Handwerk und Kleinhandel relevanten Gesetzgebung lagen - zumindest was die Intentionen der zuständigen Bundesministerien angeht - nicht primär mittelstandspolitische Motive zugrunde. Erhards wirtschaftspolitische Entscheidungen wurden in erster Linie von seinen stabilitätspolitischen Zielen be- 
stimmt. Es ging ihm insbesondere um Preisniveaustabilität und wirtschaftliches Wachstum. Um diese Ziele zu erreichen, bediente er sich mitunter sogar marktinkonformer Mittel. Diese Politik wirkte sich auf der Ebene des gewerblichen Mittelstands unterschiedlich aus. So wurde z. B. durch die Preisbindung der zweiten Hand und die Mittelstandsempfehlungen des GWB die freie Preisbildung im Kleinhandel eingeschränkt, während die Preistreibereibestimmung des Wirtschaftsstrafgesetzes die Funktion hatte, die mittelständischen Einzelhändler zu einer wettbewerbsgerechten Preisbildung zu veranlassen. Auch die Beschränkung der konsumgenossenschaftltichen Rückvergütung wurde vom Bundeswirtschaftsministerium in erster Linie aus preispolitischen Erwägungen gutgeheißen und nicht, um den traditionellen Kleinhandel vor Konkurrenz zu schützen. In der Ladenschlußfrage stellte sich das Bundeswirtschaftsministerium sogar gegen die Forderungen des mittelständischen Einzelhandels. Erhard befürchete, daß im Falle einer Verkürzung der Verkaufszeiten die Warendistribution und, damit einhergehend, die Konsummöglichkeiten eingeschränkt würden. Grundsätzlich ist zu betonen, daß Erhard kein Feind der Großbetriebe war. Seine Kritik richtete sich lediglich gegen marktbeherrschende Unternehmen. Die Expansion der Großbetriebsformen im Einzelhandel kam dem Bundeswirtschaftsminister durchaus gelegen, da diese Kosten- und Preissenkungen nach sich zog.

Die Reform der Einkommensteuergesetzgebung zielte auf die Förderung der mittleren Einkommensschichten, zu denen nicht allein die mittelständischen Selbständigen, sondern auch die Angehörigen des neuen Mittelstandes sowie ein großer Teil der Freiberufler zählten. Bundesfinanzminister Schäffer, der vor allem an der Sanierung des Bundeshaushaltes interessiert war, vertrat im Gegensatz zu Erhard die Auffassung, daß die Mittelstandsförderung in erster Linie Aufgabe der Kreditpolitik sowie der Gewerbeförderung, aber nicht der Steuerpolitik sei. Schäffer konnte jedoch nicht verhindern, daß seit Mitte der 50er Jahre die Interessen des gewerblichen Mittelstandes in der Steuergesetzgebung zunehmend berücksichtigt wurden. Sein Nachfolger Etzel bekannte sich ausdrücklich zur wirtschaftspolitischen Funktion der Steuerpolitik im allgemeinen und zur Förderung der Klein- und Mittelbetriebe im besonderen.

Für das Bundesarbeitsministerium war die Mittelstandsförderung von nachrangiger Bedeutung. Der Bundesarbeitsminister war - ebenso wie der linke Flügel der Union vornehmlich an der Verbesserung der Beschäftigungssituation und des Arbeitnehmerschutzes sowie am Ausbau der sozialen Sicherung interessiert. Dies erklärt, warum das Bundesarbeitsministerium gegen die von den Mittelstandsverbänden geforderte Herausnahme der Kleinbetriebe aus dem Geltungsbereich des Kündigungsschutzgesetzes und des Betriebsverfassungsgesetzes opponierte. Die von der Mittelstandsförderung ausgehenden Beschäftigungseffekte wurden als gering eingestuft. Soweit das Bundesarbeitsministerium in die Kreditpolitik involviert war, trat es dafür ein, die Großbetriebe bei der Kreditvergabe zu bevorzugen. Auch auf dem Gebiet des Jugendarbeitsschutzes und der Lehrlingsvergütung gingen die Anschauungen von Bundesarbeitsministerium und Mittelstandsverbänden weit auseinander. Storch stellte die Reform der Jugendarbeitsschutzvorschriften zu Beginn der 50er Jahre nur deshalb zurück, weil er die Einstellung von Lehrlingen nicht erschweren wollte. Der vom Handwerk angedrohte Ausbildungsboykott zeigte also Wirkung. Der Bundesarbeitsminister wurde erst initiativ, als sich das Ende des Lehrstellenmangels und der Berufsnot der Jugendlichen abzeichnete. Die Pflichtmitgliedschaft der selbständigen Handwerker in der gesetzlichen Rentenversiche- 
rung wurde vom Bundesarbeitsministerium unter der Voraussetzung befürwortet, daß der Versichertengemeinschaft der Angestellten bzw. Arbeiter nicht zugemutet wurde, die schlechten Risiken aus der Handwerkerversicherung zu übernehmen. Der Einfluß des Bundesarbeitsministeriums reichte indes nicht aus, um die diesbezügliche Privilegierung der Handwerker zu verhindern. $\mathrm{Zu}$ Beginn der dritten Legislaturperiode schien es zunächst so, als würden die sozialpolitischen Interessen der Klein- und Mittelbetriebe künftig stärker berücksichtigt. Es zeigte sich jedoch sehr bald, daß die von den Mittelstandsverbänden erhobene Forderung nach einer sozialpolitischen Sonderbehandlung der in Klein- und Mittelbetrieben beschäftigten Arbeitnehmer ebensowenig durchsetzbar war wie der vom $\mathrm{ZDH}$ ins Gespräch gebrachte Vorschlag, das Beitragsbemessungssystem zugunsten der mittelständischen Betriebe umzugestalten. Eine wettbewerbsneutralere Gestaltung der Sozialgesetzgebung kam für das Bundesarbeitsministerium nur in Frage, wenn hierdurch die als vorrangig erachtete sozialpolitische Förderung der abhängig Beschäftigten nicht konterkariert wurde. Der Bundeswirtschaftsminister teilte prinzipiell die Kritik der Mittelstandsverbände an der Sozialpolitik. Anfang der 60er Jahre rang er sich indes zu der Auffassung durch, daß die durch die Sozialgesetzgebung entstehenden Wettbewerbsverzerrungen zwischen Groß- und Kleinbetrieben hingenommen werden müßten. Es sei Aufgabe der Wirtschaftspolitik, diese für die ökonomische Entwicklung der Klein- und Mittelbetriebe negativen Effekte zu kompensieren.

Betrachtet man die Verbandspolitik des ZDH im Überblick, so sind in bezug auf die ideologisch-programmatische Ausrichtung wesentliche Veränderungen zu konstatieren. Anfang der 50er stand die handwerkspolitische Agitation noch ganz im Zeichen des Berufsstandsgedankens. Die besondere soziale und kulturelle Bedeutung des Handwerks wurde gepriesen, die Interessengegensätze zwischen Gesellen und Betriebsinhabern geleugnet und die Distanz gegenüber „Arbeit“ und „Kapital“ betont. Darüber hinaus vertrat die Handwerksführung die Ansicht, daß es sich bei den Handwerkern nicht um einen Kleinunternehmertyp handele. Kapitalistische Prinzipien seien mit dem Wesen des Handwerks nicht vereinbar. Die Lebens- und Geschäftschancen der Kleingewerbetreibenden, so könnte man pointiert formulieren, sollten gegen die traditionszerstörenden Wirkungen der Marktgesellschaft verteidigt werden. Das demokratische System der Bundesrepublik wurde zwar grundsätzlich anerkannt, als Gegenleistung erwartete der ZDH allerdings, daß man die Interessen der Handwerker „gebührend“ berücksichtigte. Als dies während der Gründungskrise der Bundesrepublik nicht geschah, scheute die Handwerksführung nicht davor zurück, nach dem Muster der mittelständischen Sammlungspolitik der Wilhelminischen Zeit eine Organisation ins Leben zu rufen, die zumindest in ihren Anfängen Züge einer außerparlamentarischen und tendenziell antiparlamentarischen Protestbewegung aufwies. Insgesamt wird man feststellen können, daß die handwerkliche Verbandspolitik zu Beginn der 50er Jahre in der Kontinuität der Mittelstandsideologie stand. Die ideologische Orientierung der Handwerksbasis ist zwar quellenmäßig schwerer faßbar als die des $\mathrm{ZDH}$, immerhin gibt es aber Indizien, die darauf schließen lassen, daß ein beträchtlicher Teil der selbständigen Handwerker durchaus auf der Linie der Verbandspolitik lag. Das Fortwirken der traditionellen Handwerksideologie auf verbandlicher Ebene dürfte zum einen darauf zurückzuführen sein, daß die meisten Spitzenfunktionäre ihre verbandspolitische Prägung während der Weimarer Republik erhalten hatten. Zum anderen hatte die Betonung der Berufsstandsidee die Funktion, die geforderte Berufsordnung zu legitimieren. 
Die Überzeugungskraft des Berufsstandsgedankens ließ Mitte der 50er Jahre spürbar nach. Angesichts des wirtschaftlichen und gesellschaftlichen Wandels wirkten derartige Begründungsmuster zunehmend anachronistisch. Dies erkannte auch die Handwerksführung. Die berufsständische Rhetorik trat daher in den Hintergrund. Gleichzeitig kam es zu einer konzeptionellen Neuorientierung der Verbandsspolitik. Die Förderungswürdigkeit des Handwerks wurde nicht länger mit der soziokulturellen Sonderstellung dieser Berufgruppe, sondern mit marktkonformen wettbewerbspolitischen Argumenten begründet. Der ZDH beschränkte sich mehr und mehr darauf, die Wettbewerbsneutralität zwischen Klein- und Großbetrieben einzufordern. Sozialprotektionistische Motive lassen sich zwar auch noch gegen Ende des Untersuchungszeitraums nachweisen, ihr Stellenwert nahm jedoch in der zweiten Hälfte der 50er Jahre ab. Der $\mathrm{ZDH}$ sah es in zunehmendem Maße als seine Aufgabe an, den Handwerksbetrieben die Anpassung an die wirtschaftlichen Rahmenbedingungen zu erleichtern. Die Handwerksbetriebe sollten modernisiert und der Unternehmergeist der Betriebsinhaber gefördert werden. Die mit dem Strukturwandel einhergehende Schrumpfung des Betriebsbestandes wurde dabei ebenso in Kauf genommen wie der branchenstrukturelle Wandel, der insbesondere zu Lasten der traditionellen Handwerkszweige ging. Dies fiel insofern nicht schwer, als diese strukturellen Veränderungen zur Stabilisierung der Handwerkswirtschaft beitrugen. Dem neuen politischen Kurs des $\mathrm{ZDH}$ wurde von der Handwerksbasis kein nennenswerter Widerstand entgegengesetzt. Der Abbau des Radikalisierungspotentials ist vor allem damit zu erklären, daß sich die ökonomische und soziale Lage eines Großteils der Handwerker verbesserte. Des weiteren dürfte eine Rolle gespielt haben, daß die Forderungen des Handwerks zunehmend Berücksichtigung fanden. Die Abkehr des ZDH von der Status-Quo-Potitik wirkte sich auf die Zusammenarbeit mit der Bundesregierung positiv aus. Die Handwerksführung und das Bundeswirtschaftsministerium zogen jetzt in zentralen handwerkspolitischen Fragen am gleichen Strang.

Ende der 50er Jahre setzte der ZDH auch in der Mittelstandspolitik neue Akzente. Die Handwerksführung vertrat nunmehr das Konzept einer „mittelstandsbetonten Strukturpolitik ${ }^{c}$, das darauf abzielte, angemessene Wettbewerbsbedingungen für Kleinund Mittelbetriebe in Industrie, Handwerk und Handel zu schaffen. Intendiert war also eine betriebsgrößenorientierte Kleinunternehmerpolitik, in deren Rahmen die berufsgruppenspezifischen Anliegen nur von sekundärer Bedeutung sein sollten. Die Bereitschaft des Handwerks, mit der Kleinindustrie eine interessenpolitische Koalition zu bilden, ist nicht zuletzt vor dem Hintergrund der wachsenden Interessengegensätze zwischen industriellen Großbetrieben und mittelständischen Industrie- und Handwerksbetrieben zu sehen. In den industriellen Großunternehmen wuchsen die freiwilligen Sozialaufwendungen in den 50er Jahren rasch an. Dies erleichterte die Rekrutierung von Arbeitskräften. Die Klein- und Mittelbetriebe, die von dem Mitte der 50er Jahre einsetzenden Fachkräftemangel besonders stark betroffen waren, fühlten sich zudem durch die tarifpolitischen Vereinbarungen, die sich an den leistungsstärkeren Betrieben orientierten, benachteiligt. Darüber hinaus hielten es die Interessenvertretungsorgane der Klein- und Mittelbetriebe gegen Ende des Untersuchungszeitraums für notwendig, dem wirtschaftlichen Konzentrationsprozeß Einhalt zu gebieten.

Die Einzelhandelsführung distanzierte sich nach 1945 zunächst von der Mittelstandsideologie der Weimarer Zeit. Hans Schmitz und Wilhelm Naegel, die beiden führenden 
Köpfe der HDE, bemühten sich gegenüber den Einzelhandelsgroßbetrieben, den Konsumgenossenschaften und den Gewerkschaften um eine Politik des Ausgleichs. Sie gerieten dabei zunehmend in Widerspruch zur mittelständischen Einzelhandelsbasis. Der Versuch, die Kritiker davon zu überzeugen, daß der beste Schutz der Kleinbetriebe die betriebliche Rationalisierung sei, schlug fehl. Ende 1951 sah sich die HDE-Führung genötigt, den protektionistischen Forderungen der mittelständischen Betriebsinhaber nachzugeben. Das erste greifbare Ergebnis dieses verbandspolitischen Kurswechsels war das Expansionsstoppabkommen vom Sommer 1952, in dem sich die Interessenvertreter der Warenhäuser und Filialbetriebe verpflichteten, ihre Mitgliedsbetriebe zu einem vorübergehenden Expansionsverzicht anzuhalten. Im Gegenzug versprach die HDE, die Mittel- und Großbetriebe vor Diskriminierung zu schützen. Wie sich bald herausstellte, ging das Abkommen den sich immer stärker radikalisierenden Kleinhändlern nicht weit genug. Die HDE, die immer mehr unter den Einfluß der Mittelstandsvertreter geriet, forderte daraufhin den Gesetzgeber auf, objektive Zulassungsbeschränkungen für Einzelhandelsgroßbetriebe einzuführen. Das Bundeswirtschaftsministerium wies dieses wettbewerbsfeindliche Ansinnen zurück. Dieser Vorstoß der HDE, der allein aus verfassungsrechtlichen Gründen von vonherein zum Scheitern verurteilt war, bewirkte, daß die Einzelhandelsführung von der mittelständischen Basis als legitimes Interessenvertretungsorgan anerkannt wurde. Den gleichen Effekt hatte der im Frühjahr 1953 erfolgende Beitritt der HDE zum Mittelstandsblock. Ende der 50er Jahre verschärfte sich der Protest der mittelständischen Betriebsinhaber gegen die einzelhandelsinterne Konzentration. Die Kritik richtete sich jetzt nicht mehr allein gegen Warenhäuser und Filialbetriebe, sondern darüber hinaus gegen die neuen Konzentrationsformen wie z. B. die Supermärkte. Insbesondere der Lebensmitteleinzelhandel, in dem die Radikalisierungsneigung traditionellerweise besonders stark ausgeprägt war, fühlte sich durch das Auftreten dieser hochrationalisierten Betriebsformen bedroht. Erneut gab die Einzelhandelsführung dem Drängen der Basis nach und setzte sich beim Bundeswirtschaftsministerium dafür ein, die Ausdehnung der Supermärkte staatlicherseits zu stoppen. Die HDE konnte sich indes mit dieser Forderung nicht durchsetzen.

Vergleicht man die verbandspolitische Entwicklung in Handwerk und Einzelhandel, so ist festzustellen, daß die HDE länger an mittelstandsprotektionistischen Positionen festhielt als der ZDH. Gleichwohl wäre es verfehlt, anzunehmen, es sei der Einzelhandelsführung ausschließlich um die Konservierung bestehender Strukturen gegangen. So förderte die HDE bereits zu einem recht frühen Zeitpunkt die Rationalisierung der Einzelhandelsbetriebe. Die HDE-Führung stellte sich auch nicht gegen die Expansion der genossenschaftlichen Großzusammenschlüsse, obgleich diese den unternehmerischen Dispositionsspielraum der Kleinhändler einschränkten. Dies ist vor allem damit zu erklären, daß die HDE in der genossenschaftlichen Selbsthilfe ein unverzichtbares Mittel im Kampf gegen die zunehmende Konzentration sah. Insgesamt wird man feststellen können, daß sich in den 50er Jahren in verbandspolitischer Hinsicht ein Modernisierungsschub im gewerblichen Mittelstand vollzog, der durch die zunehmende Akzeptanz marktwirtschaftlicher Prinzipien und Erfordernisse gekennzeichnet war. 